\title{
Usefulness of Mapping Biopsy in the Treatment of Penoscrotal Extramammary Paget's Disease
}

\author{
Curtis A. Pettaway, MD \\ The University of Texas, M.D. Anderson Cancer Center, Houston, TX
}

Park et al. report their experience in the management of extramammary Paget's Disease (EMPD) of the penoscrotal region and specifically compare outcomes among cohorts of men with the disease who either did or did not undergo mapping biopsies prior to their definitive surgical procedure. The rationale for the study and this comparison is that Paget's disease initially spreads insidiously through the epidermis, sometimes in a single-cell fashion, and establishing the diagnosis can be very difficult subsequent to intraoperative frozen sections. Thus, several studies have described the use of outpatient mapping biopsies under more permanent section pathology techniques to facilitate the diagnosis and to 'clear' the surgical margins (references 19-21 in the article). This should theoretically lead to a lower incidence of positive frozen section margins intraoperatively, a lower incidence of positive permanent section margins, and lower recurrence rates for patients.

The main inconvenience for patients with mapping biopsies is that they may have to undergo a number of outpatient procedures prior to the definitive surgical procedure. In the current study, 44 patients were evaluated, with 26 undergoing mapping biopsies and 18 having standard intraoperative wide excision starting $2 \mathrm{~cm}$ beyond the gross lesion. All patients left the operating room with negative frozen section margins. As expected, the mapping biopsy group had a significantly lower incidence of positive frozen section margins (61 vs. $15.4 \%$ ), a lower rate of positive permanent margins (30.8 vs. $61.1 \%$ ), and a lower incidence of local recurrence (11.5 vs. $50 \%$ ) than patients who did not undergo mapping biopsies. The authors conclude that these data support their conclusions

\section{(C) Society of Surgical Oncology 2017}

First Received: 10 June 2017;

Published Online: 20 June 2017

C. A. Pettaway, MD

e-mail: cpettawa@mdanderson.org about the efficacy of mapping biopsies in their hands, which is true. However, there are several ways I believe patient care can be improved in the setting of the surgical management of patients with penoscrotal EMPD. First, it is noteworthy that in their study, despite all patients leaving the operating room with negative surgical margins, 30.8 and $61.1 \%$ of patients with and without mapping biopsies, respectively, still had positive permanent section disease at their surgical margins. Thus, they must also conclude that the general pathologists at their center were unable to provide a frozen section diagnosis of EMPD in many cases. This can be overcome in several ways. (1) We routinely utilize dermatopathology specialists to analyze our frozen section margins and have reported no recurrences among patients with intraepidermal Paget's' disease. ${ }^{1}$ Another group from Korea has also reported only a $7 \%$ rate of permanent section margins after negative frozen section margins among their EMPD cohort. $^{2}$ (2) Trained Moh's surgeons also possess the pathologic expertise to diagnose and manage these patients, utilizing outpatient surgical techniques with low recurrence rates (reference 16 in the article). (3) Immunohistochemical techniques have been developed to facilitate making the diagnosis intraoperatively on frozen section (reference 25 in the article). Thus, it is my opinion that EMPD patients should be referred to centers with not only the surgical expertise but also the accompanying pathologic expertise necessary to diagnose subtle pathologic changes to optimize perioperative decision making. In such settings, wide local excision and Mohs surgery can both be effective in managing such patients with or without prior mapping biopsies.

\section{REFERENCES}

1. Hegarty PK, Suh J, Fisher MB, et al. Penoscrotal extramammary Paget's disease: the University of Texas M. D. Anderson Cancer Center contemporary experience. J Urol. 2011;186:97-102

2. Yang WJ, Kim, DS, Im, YJ, et al. Extramammary Paget's disease of penis and scrotum. Urology. 2005;65 (5):972-975 